\title{
El espíritu de nuestro tiempo: las incertidumbres y posibilidades del trabajo en la educación básica
}

Rivera Alfaro, Ronald; González Conejo, Franky

El espíritu de nuestro tiempo: las incertidumbres y posibilidades del trabajo en la educación básica

Revista Educación, vol. 45, núm. 1, 2021

Universidad de Costa Rica, Costa Rica

Disponible en: http://www.redalyc.org/articulo.oa?id=44064134015

DOI: https://doi.org/10.15517/revedu.v45i1.40843

Esta obra está bajo una Licencia Creative Commons Atribución-NoComercial-SinDerivar 3.0 Internacional. 


\title{
El espíritu de nuestro tiempo: las incertidumbres y posibilidades del trabajo en la educación básica
}

\author{
Ronald Rivera Alfaro \\ Universidad Nacional, Costa Rica \\ ronald.rivera.alfaro@una.ac.cr \\ (iD http://orcid.org/0000-0001-9179-3517 \\ Franky González Conejo \\ Costa Rica, Universidad Nacional., Costa Rica \\ franky.gonzalez.conejo@una.cr \\ iD http://orcid.org/0000-0002-9991-8078
}

The Spirit of our Times: The Uncertainties and Possibilities of Working in Primary Education

\author{
DOI: https://doi.org/10.15517/revedu.v45i1.40843 \\ DOI: https://doi.org/10.15517/revedu.v45i1.40843
Redalyc: http://www.redalyc.org/articulo.oa?id=44064134015
}

Redale: het//www.redalyc.org/articulo.02in=44064134015

\section{(1)}


aunque falible en su concepción metodológica sobre el comportamiento racional y de evolución cognitiva, permite evidenciar las contradicciones y dislocaciones que como sociedad mantenemos y no asumimos, al colocar en riesgo nuestras condiciones de vida.

En este sentido, se discute sobre el trabajo como categoría central para el desarrollo de la sociedad, especialmente en el ámbito de la educación básica, donde se presentan altos niveles de subempleo y bajos salarios, en contraste con altos grados de satisfacción de las docentes por la formación recibida.

En consecuencia, la tasa de desempleo profesional en Costa Rica está en constante crecimiento, pero aún es menor $^{[1]}$ que la tasa de desempleo abierto a nivel general. Al menos en las disciplinas de la educación básica, estas tasas son más elevadas que en otras áreas profesionales, sumado al bajo promedio salarial con respecto a otras áreas de educación. Este problema que interpela directamente en el buen ejercicio docente será asumido y analizado en concordancia con un enfoque amplio y ético del trabajo.

Se parte de la revisión y análisis de investigaciones y datos del contexto sociolaboral de la educación básica en Costa Rica, particularmente de las carreras pertenecientes a la Universidad Nacional, elaborados, en su mayoría, por el Observatorio Laboratorio de Profesiones (OLaP) del Consejo Nacional de Rectores (CONARE); posterior a esto se analizan los datos desde una reflexión conceptual y crítica del trabajo bajo la motivación del Espíritu de nuestro tiempo, obra dadaísta que amarra las contradicciones y el sinsentido del mundo de trabajo asentadas en las desigualdades salariales y de reconocimiento social.

Por tal motivo, además del volumen de datos que está disponible en los Informes de la Encuesta Continua de Empleo desarrollados por el Instituto Nacional de Estadística y Censo (INEC), la ruptura y novedad en el análisis se concierta desde su concepción como problema, para ello se utiliza la obra El espíritu de nuestro tiempo de Raoul Hausmann (1919), artista austriaco, la cual permite reflexionar sobre el control y la manipulación en un mundo mecanizado, colapsado, dirigido por estructuras tecnificadas aplomadas en una racionalidad social representada por una cabeza humana inexpresiva, que no escucha, que no ve, cuyo enlace con la mecanización de la educación y su relación, desigual y marginal, con el mercado laboral, es elocuente.

Dicha obra hace reseña del clima moral, intelectual, económico y cultural de una época, es metáfora que cuestiona otros perfiles de otras épocas en alusión a la disertación hegeliana de las condiciones humanas que retrata Hausmann, y que noventa años después siguen encontrando sentido de realidad.

El Zeitgeist o el espiritu de una época se establece bajo la referencia a la materialidad concebida desde el diálogo, y ese diálogo encarnado en el trabajo, cuyo acervo se asienta en (1) el deterioro de las condiciones laborales, (2) la escasez del empleo y (3) en el empobrecimiento o poco valor cultural al desarrollo y ejercicio de disciplinas y profesiones que influyen en el pensamiento ciudadano; precisamente las que presentan indicadores de inserción laboral menos favorables.

En este sentido, la educación como eje del pensamiento político-intelectual que sostiene e impulsa a los Estados a mantener vivas sus conciencias políticas (realidad social y acción ciudadana para el desenvolvimiento del sistema democrático) y nacionales (actitudes cívicas y resguardo de los valores patrios), ha sido paulatinamente instrumentalizada por la reconversión tecnológica-financiera que el capitalismo ha instituido y consolidado por sus múltiples crisis económicas, a tal punto de convertirse en un argumento político por recortar los presupuestos en educación y justificar el trabajo técnico, como bien lo dijera Carlos Alvarado, presidente de Costa Rica, en julio del 2019, en la inauguración de un campus privado, "cada vez menos los títulos universitarios tienen importancia en el mundo de hoy" (Arrieta, 2019, párr. 4).

Esta perspectiva política augura una mecanización y estratificación de los saberes en habilidades y certificaciones, lo que valida un modelo de desarrollo fundamentado en la capacitación continua; por eso se logra identificar la base material que sostendría un "proceso sociometabólico del capitalismo" (Grüner, 2011, p. 16), el cual subyace en la lógica matricial del fetichismo de la mercancía, alude a Mészàros (2002) y los avatares del pensamiento crítico, aplicable a la educación reconvertida como trabajo, no como derecho público social ${ }^{[2]}$ o posibilidad de transformación social. 
Este sesgo, que solo mira la empleabilidad y viabilidad de la educación desde su incidencia en los mercados actuales, desecha todo aquello que no dé resultados favorables dentro de la lógica de operación financiera, es decir, la demanda determina la oferta educativa en un mundo globalizado y mercantilizado.

Para iniciar esta descontractura de la industria educativa, como bastión o soporte de las nuevas fuerzas de trabajo inducidas al mercado educativo, incluidas las personas docentes, académicas y especialistas, es necesario dar una breve mirada a la ética del trabajo, la justicia social y el deber moral de cumplir con el principio de reciprocidad y comunidad que tiene la educación pública.

\section{ACERCA DE LA ÉTICA DEL TRABAJo}

Desde la elucubración de Hesíodo, en la Grecia antigua, al referirse al mundo del trabajo como una virtud, las circunstancias que se crean y conectan en las actividades que definen al trabajo como actividad económica, obligan a repensar las expectativas, deseos, vicios y vacíos que acarrean las nuevas formas de trabajo en el siglo XXI, puesto que no siempre el trabajo con fin económico ha sido la actividad humana dominante, puesta en advertencia hace casi treinta años por Andre Gorz (1997) en su Metamorfosis del trabajo, y ahora constituida como uno de los principales retos de la política y la economía.

La centralidad del trabajo productivo es dominante a partir de la llegada del capitalismo industrial, y con ello la disyuntiva entre la actividad desarrollada, su valor real en el intercambio de bienes, el salario recibido, el tiempo empleado y la satisfacción de la tarea ejecutada. Todo lo anterior focaliza el falso sentido de la vida mediante la sobrevivencia por tener un empleo, lo que se constituye como el principal ingreso de toda la familia, y el determinante absurdo ${ }^{[3]}$ para definir una vida exitosa; opuesto a la satisfacción por sentido de vocación, que otorga el valor central del trabajo, principalmente en la vida de la persona adulta como expone Rivas (2003).

En Costa Rica, según la tercera encuesta trimestral de empleo de 2019, de la totalidad de la población, el $45 \%$ se ubica dentro de la categoría fuerza de trabajo, es decir, personas con capacidades para desarrollar actividades productivas, y de ese total, el 11,4\% están desempleadas, sin premoniciones de que esta situación mejore para el resto del año, ya que el Instituto Nacional de Estadística y Censo (INEC) estimó en 278 mil las personas desempleadas y otras 36 mil personas más buscaron activamente un empleo y no lo encontraron (INEC, 2019). A esto se le suma un 11,6\% de la población ocupada en condiciones de subempleo y, aunque cuentan con la posibilidad de retornar al trabajo y recibir un sueldo, la jornada laboral puede ser inferior a las 40 horas semanales y con ello una relativización del ingreso y oportunidad de compra de bienes para la subsistencia.

Según el Banco Mundial, la lógica de rentabilidad del mercado hará que entre el 10\% y 60\% de empleos actuales sean proclives a desaparecer o ser sustituidos, con el sesgo de enfocarse exclusivamente en la viabilidad técnica de sustitución. Así, "se prevé que desaparecerán por completo menos del 20 por ciento de los empleos” (Banco Mundial, 2017, p. 23), no obstante, la labor educativa en clave de mediación pedagógica no es una labor que se vea en peligro por la tecnificación y estandarización de los procesos.

En un estudio elaborado por el Centro de Implementación de Políticas Públicas para la Equidad y el Crecimiento (CIPPEC) (Albrieu, Brest, Larroulet, Rapetti y Sorrentino, 2019), subsidiado por Microsoft Latinoamérica, Inteligencia artificial y crecimiento económico. Oportunidades y desafíos para Costa Rica, se establece el rol práctico de la educación básica según la metodología de escenarios utilizada y coloca, como tarea central, la educación en la primera infancia, esto debido a la formación de las bases estructurales del cerebro, "de manera que constituye un período clave para desarrollar habilidades cognitivas y socioemocionales básicas [...] es necesario mejorar la calidad de la educación básica para poder incorporar habilidades y conocimientos generales más avanzados” (p. 25).

Asimismo, coloca entre las 10 profesiones menos susceptibles de ser automatizadas o suplidas por la Inteligencia Artificial a cuatro profesiones vinculadas directamente en el ámbito educativo: profesores de 
enseñanza secundaria, profesionales de nivel medio de la enseñanza, directores de servicios de educación y especialistas en métodos pedagógicos. Esto constata la importancia de la educación en cualquier modelo de desarrollo, y la coloca como categoría central del desarrollo.

Por tal razón, desde una perspectiva ampliada del trabajo, la educación costarricense sigue la consigna elemental de la Organización Mundial del Trabajo, al definir que el trabajo no puede ser tratado como una mercancía que se intercambia en los mercados laborales altamente segmentados. Si bien las personas profesionales deben tener mejores oportunidades laborales, no se eximen de los problemas que se presentan a la hora de insertarse, por primera vez, al mundo laboral. Para Teichler (2005), Enriques (2007) y Echeverría, Isus, Martínez y Sarasola (2008) se debe a una oferta excesiva de profesionales universitarios (en muchos casos con un perfil semejante), con carencia de competencias necesarias que no se exigían anteriormente, como las relacionadas con la autogestión, y la comunicación que tanto valora el sector empresarial, consecuente con las nuevas formas de laborar y con los modelos económicos que imperan en la mayoría de los países latinoamericanos.

Como evidencia de la sobreoferta de profesionales en el contexto universitario costarricense, según el VII Informe del Estado de la Educación (Programa Estado de la Nación, 2019) entre 2012 y 2017 se entregaron 46.800 títulos como promedio cada año, y a pesar de que la cantidad de graduados en el área de Educación viene a la baja desde el año 2000, actualmente el 20\% de la oferta académica (la mayoría) corresponde a carreras de enseñanza. Además, del mismo informe se desprende que las universidades privadas aportan buena parte de la sobreoferta de educadores, pues para las siete que más gradúan, el $40 \%$ de sus carreras son del área de Educación, con perfiles profesionales desactualizados en su mayoría, si se considera que, a nivel nacional el 62,7\% de los planes de estudio en Educación que abrieron hace más de 10 años, nunca han sido modificados parcial, ni sustancialmente, aunque sigue habiendo mayor renovación de la oferta en las universidades estatales.

Por otro lado, se ratifica una considerable diferencia en cuanto a las posibilidades de insertarse laboralmente dentro o fuera de la zona metropolitana, con espacios de trabajo ya saturados o con opciones poco compatibles con los perfiles profesionales, es por ello que la gran mayoría decide quedarse trabajando en el centro del territorio nacional, que les ofrece mayores oportunidades laborales y de continuar estudios, principalmente en San José, como lo confirmó en un segundo estudio consecutivo el Observatorio Laboral de Profesiones (OLaP-Conare) en Gutiérrez, Kikut, Corrales y Picado (2018), escenario que no aporta al desarrollo económico y social de las zonas rurales, a pesar de las diferentes sedes y campus universitarios en todo el país.

A pesar de las fortalezas que se reconocen en una persona titulada de universidad estatal, la situación descrita puede hacer que esta experimente incertidumbre en dos vías, la primera relacionada con el desconocimiento de las oportunidades que le brinda el mercado laboral de su carrera y las expectativas que tiene con respecto a ejercer su profesión; la segunda, debido a la falta de experiencia laboral, común en la gran mayoría de los recién graduados, quienes identificaron este aspecto, junto con la baja demanda laboral en su campo, como los dos principales motivos por los que no consiguen trabajo (Gutiérrez et al., 2018).

En consecuencia, tenemos un panorama poco alentador para las personas recién graduadas o que se encuentran estudiando aún carreras de educación, en el sentido de que la demanda laboral — con el Ministerio de Educación Pública (MEP) como principal empleador - no está absorbiendo o no es compatible con la sobreoferta profesional que se diseña curricularmente en las universidades, por lo que se vuelve urgente que las facultades de educación tengan una vinculación más estrecha con las organizaciones del mundo del trabajo, como son los principales empleadores, los colegios profesionales y, en el caso de Costa Rica, la Dirección General de Servicio Civil, que se encarga de desarrollar todo el proceso de intermediación y selección que requiere el MEP para contratar; de igual forma, explorar ámbitos laborales no tradicionales para la persona profesional docente, en los cuales incluso ya podrían existir experiencias valiosas de los propios graduados. 


\section{EL TRABAJO EN LA EDUCACIÓN BÁSICA}

En este contexto, la educación básica la componen las disciplinas relacionadas con la instrucción y mediación pedagógicas en las edades tempranas, desde el nacimiento hasta los 12 años, incluidos los procesos de alfabetización que abarcan otras poblaciones, y la educación especial. En el caso de la Universidad Nacional de Costa Rica, dicha área incluye las siguientes carreras: Bachillerato en Enseñanza del Inglés para I y II Ciclos, Bachillerato y Licenciatura en Educación Especial, Bachillerato y Licenciatura en Pedagogía con énfasis en Educación Preescolar y Bachillerato y Licenciatura en Pedagogía con énfasis en I y II Ciclos de la Educación General Básica.

Con respecto al mundo laboral, y en particular de estas carreras, se extraen algunos datos importantes del informe que preparó el OLaP para la Universidad Nacional (Gutiérrez, Corrales y Sandí, 2019). En el siguiente cuadro, se presenta el comparativo con los indicadores del área de Educación como parámetro base.

TABLA 1

Porcentaje de personas graduadas en el período 2011-2013, de cuatro disciplinas de la Educación Básica, según indicador laboral al 2016

\begin{tabular}{|l|l|l|l|l|}
\hline Área/Disciplina & \% Desempleo & $\begin{array}{l}\text { \% Subempleo } \\
\text { por horas }\end{array}$ & $\begin{array}{l}\text { \% Poca relación con la } \\
\text { carrera que estudia }\end{array}$ & $\begin{array}{l}\text { Tasa de presión } \\
\text { general }^{*}\end{array}$ \\
\hline Área de Educación & 6,0 & 7,9 & 2,9 & 24,8 \\
\hline Educación Primaria & 2,8 & 0,7 & 0,0 & 13,8 \\
\hline Educación Primaria Inglés & 3,3 & 10,3 & 6,9 & 25,9 \\
\hline Educación Preescolar & 9,5 & 8,8 & 0,0 & 38.8 \\
\hline Educación Especial & 4,9 & 8,9 & 0,0 & 33,7 \\
\hline
\end{tabular}

Fuente: Elaboración propia con datos de Gutiérrez et al. (2019).

* Representa a las personas desempleadas y las que trabajan pero están dispuestas a cambiar de empleo

En términos generales, como se menciona en la Tabla 1, se resalta que en Educación Primaria se presentan los mejores indicadores, todos por debajo del promedio general en el área de Educación, lo cual podría justificar que tenga los puntajes más altos con respecto al grado de satisfacción con el trabajo $(4,5)$ y con la carrera $(4,1)$, en una escala de 1 a 5 según el cuadro 2.

Además, sobresale el caso de Educación Primaria Inglés, con los indicadores más altos en subempleo $(10,3 \%)$ y poca relación entre carrera y trabajo actual $(6,9 \%)$, lo que podría motivar a que más de una cuarta parte de las personas considere cambiar de empleo.

La disciplina de Educación Especial se ubica de segunda en cuanto al porcentaje de desempleo $(4,9)$, subempleo $(8,9)$ y tasa de presión general $(33,7)$, con más de una tercera parte de personas que podría estar reflexionando sobre un cambio de trabajo, situación que pudiese tener algún efecto en el grado de satisfacción que tienen con su carrera $(3,8)$, el más bajo de las cuatro disciplinas.

En este sentido, tomando como referencia los datos, el trabajo en la educación básica es una actividad con altas tasas de subempleo, lo que acarrea precarización e inestabilidad económica y emocional, pues se valora la prospectiva de empleo - económica y socioeducativa- en relación con el tiempo para la formación invertida y el salario percibido. Asimismo, y bajo la lógica clásica de la relación capital-trabajo, la transición de la Universidad al trabajo es compleja, y está permeada por la alta oferta ${ }^{[4]}$ laboral, sumándole la utilidad del aprendizaje según los parámetros del mercado. Este segundo criterio es nefasto y recurrente, ya que supone una subsunción de la dinámica social al desarrollo capitalista, agravada por las crisis fiscales y la dependencia de las rentas del Estado a la dinámica productiva privada.

Esta situación laboral, al ser consecuencia de los procesos de desigualdad y desarrollo del país, se basa en un dualismo simplista y práctico que determina la orientación vocacional y el tipo de instrucción que se 
requieren, bajo los modelos de captación y generación de empleo del sector privado, desmeritan el sentido histórico de la educación como agente de cambio social, y sobresalta al mercado como guía y promotor del desarrollo, pues condiciona la efectividad de los programas de estudios y los saberes, habilidades y destrezas de las personas graduadas a la demanda laboral.

Esta visión, aunque deficitaria, es la que en la actualidad rige el pensamiento político de Costa Rica, el cual congrega el enfoque global de la Organización Mundial del Trabajo (OIT, 2019), en su informe Perspectivas sociales y del empleo en el mundo: Tendencias 2019, que alude a una tasa del $18 \%$ de desocupación en jóvenes, y de los que se encuentran trabajando, más del 48\% está en condiciones desfavorables en América Latina y el Caribe. Lo anterior en el contexto neoliberal y de vínculos contractuales entre las políticas sobre empleo, la seguridad social y el desarrollo económico, fundamentadas esencialmente en la tradición liberal y contractualista, las cuales superponen la acción estatal a la legitimidad del sistema económico, de libre mercado y de defensa del desarrollo mercantil como soluciones ineluctables.

TABLA 2

Grado de satisfacción con la carrera, la universidad y el trabajo de personas graduadas en el período 2011-2013, de cuatro disciplinas de la Educación Básica al 2016

\begin{tabular}{|l|l|l|l|}
\hline Área/Disciplina & $\begin{array}{l}\text { Satisfacción con } \\
\text { Su carrera }\end{array}$ & $\begin{array}{l}\text { Satisfacción con } \\
\text { universidad }\end{array}$ & $\begin{array}{l}\text { Satisfacción con } \\
\text { trabajo actual }\end{array}$ \\
\hline General Área de Educación & 4,6 & 4,4 & 4,4 \\
\hline Educación Primaria & 4,1 & 4,6 & 4,5 \\
\hline Educación Primaria Inglés & 4,0 & 4,9 & 4,3 \\
\hline Educación Preescolar & 3,8 & 4,4 & 4,1 \\
\hline Educación Especial & 3,8 & 4,6 & 4,4 \\
\hline
\end{tabular}

Fuente: Elaboración propia con datos de Gutiérrez et al. (2019).

Adicionalmente, en la Tabla 2 se logra determinar una constante: en todos los casos el grado de satisfacción con la universidad siempre es mayor a la satisfacción con el trabajo actual, y este, a su vez, mayor que la satisfacción con la carrera de la cual se titularon.

En general, los datos también revelan que las personas graduadas en el área de Educación ostentan los índices más altos de satisfacción; en primer lugar, en satisfacción con la carrera $(4,6)$ y con la universidad $(4,4)$, así como un segundo puesto en satisfacción laboral $(4,4)$; todo ello a pesar de tener carreras entre los salarios más bajos (reportados por los propios graduados según OLaP), con el mayor porcentaje de interinos $57,0 \%$, y con la menor cantidad promedio de beneficios adicionales al salario (3,5 por persona trabajadora).

Lo anterior, pone en tela de duda la correlación que hace el propio OLaP (Gutiérrez et al., 2019), al diagnosticar que "se logra evidenciar que, a mayor cantidad de beneficios mayor satisfacción con su trabajo cuentan las personas graduadas" (p. 66), por lo que se podría pensar que, para las y los profesionales en Educación, cobra mayor valor su vocación, el aporte que sienten hacer a la sociedad, su ética profesionalpersonal, entre otros elementos intangibles, que la cuantificación de los beneficios propios, como anualidades, dedicación exclusiva o pago de prohibición, entre los más citados.

Ahora bien, la educación como derecho está protegido de forma general por los Convenios Internacionales de Derechos Humanos, artículo 26 de la Declaración Universal de los Derechos Humanos (Organización de las Naciones Unidas [ONU], 1948), artículo 26 de la Convención Americana sobre Derechos Humanos (Pacto de San José) (Organización de los Estados Americanos [OEA], 1969), artículos 28 y 29 de la Convención sobre los Derechos del Niño (Asamblea General de Naciones Unidas, 1989), y artículos 77 y 78 de la Constitución Política de la República de Costa Rica (Asamblea Nacional Constituyente, 1949), incluida desde el 2018, la obligatoriedad y gratuidad de la educación preescolar para ingresar a primaria; sin embargo, la realidad dicta desigualdad y poca motivación para ejercer. $\mathrm{Al}$ menos desde el punto de vista salarial, el promedio de 
ingresos es tres veces menor al salario más alto reportado por las propias personas graduadas en el estudio del OLaP, (Gutiérrez et al., 2019), donde Preescolar es la carrera con menor ingreso con $\$ 455.048$, seguido de Educación Primaria con un promedio de $\$ 557.506$.

Lo anterior da como contexto que se quiera más y mejor educación, pero a una menor inversión. Se legisla con base en la oportunidad presentada y no en la creación de oportunidad, y esto en un país cada año más desigual subvierte el sentido real del trabajo en educación, y lo convierte en un agente más de desigualdad.

De este modo, no podemos seguir caminando por la misma vereda que da como resultado un estancamiento del desempleo, la pobreza y la desigualdad, al menos se debe forjar un nuevo trazo que regionalice y territorialice el empleo, que permita contextualizar y potencializar, en su justa medida, el trabajo, y con ello recuperar la centralidad que merece en el sentido de la vida.

\section{CONCLUSIONES Y RECOMENDACIONES}

Del análisis desarrollado, se destaca como una de las conclusiones más relevantes, las desigualdades importantes entre las condiciones laborales a las cuales acceden las personas profesionales de Educación y las que visualizan en otras áreas de conocimiento. Esto debido a la sobreoferta de educadores titulados principalmente de universidades privadas, con perfiles desactualizados incompatibles con los requerimientos del mundo laboral actual, y que se ubican en espacios saturados principalmente en la centralidad del país.

Todo este panorama de desfasaje entre formación universitaria versus ejercicio profesional, genera no solamente una competitividad laboral deshumanizante, sino una constante de incertidumbres propias del mercado de profesionales, como el absurdo de exigir experiencia laboral a personas recién graduadas.

Desde este hecho y la perspectiva de ética del trabajo del espíritu de nuestro tiempo, las personas profesionales en educación básica ven limitadas sus condiciones y expectativas laborales como actores sociales, sin que se retome el potencial creativo de transformación de la educación, y se subsuma su labor a la relación con el mercado y la oferta laboral. En esta reorganización del mundo del trabajo, como proceso histórico inacabado, impulsa y determina el trabajo como una mercancía, en contraposición de la filosofía de la OIT y el trabajo decente.

Asimismo, se concluye que a pesar de ser la educación básica una de las profesiones con menor rango salarial percibido y mayor precarización laboral, exhibe altos índices de satisfacción vocacional-laboral, pues la centralidad del trabajo y su valoración no se da en términos monetarios, más bien, desde una ética que atesora su impacto en la conformación del tejido social, mediante el favorecimiento de las capacidades de aprender a aprender y de socialización.

Por otra parte, debido a la brecha entre la oferta de profesionales y la demanda de talento cualificado, así como el aumento de oferentes con perfiles muy similares (sobre todo que se titulan de universidades privadas) y la incapacidad del país para crear nuevos puestos de trabajo, el decrecimiento sostenido del sector servicios y turismo - el más importante para el grupo de profesionales, existen cada vez menos posibilidades de afrontar las limitaciones del mercado laboral en el sector educativo. Al respecto, las carreras de educación urgen de explorar campos laborales emergentes, como es el ejercicio liberal de la profesión desde una perspectiva de capacitación de personas adultas, por ejemplo, para favorecer procesos de aprendizaje con sus hijas e hijos, conocer las experiencias de las personas graduadas en espacios no tradicionales de docencia, podrían ampliar este crisol, incluso para su posterior incorporación en los planes de estudio.

La adecuación con el área de estudio escogida por el estudiantado y la requerida en el puesto de trabajo es un factor determinante de la posterior satisfacción de las personas egresadas con sus trabajos. Asimismo, este indicador se asocia a otros acerca de la saturación del mercado laboral y las condiciones de los trabajos a quienes pueden acceder los egresados de una u otra área de estudio.

Esta adecuación entre la carrera y el puesto de trabajo es lo que permite el ajuste vocacional-laboral al cual se refiere Rivas (2003), además de la calidad o las condiciones del empleo al que logra acceder, determinadas por 
las posibilidades que ofrece o condiciona el mundo laboral de su profesión, en un escenario tan competitivo e incierto como es el global, con un camino minado de indicadores de desempleo, tasa de presión general, subempleo y poca relación entre el trabajo y la carrera que estudiaron (González, 2019).

Asimismo, es necesario resolver problemas relacionados con situaciones de inseguridad e incertidumbre provocadas por la flexibilización de las formas de contratación, el progreso tecnológico y la exigencia de nuevas competencias profesionales. Esto en palabras de Rocabert (s.f.) citado en Rivas (2003), es orientar en la flexibilidad para el cambio y la incertidumbre contextual, mediante el fortalecimiento curricular de competencias de adaptación para el desarrollo a lo largo de la vida, lo que demanda de las personas universitarias ciertas exigencias que su educación universitaria no cumple a cabalidad, por ejemplo, el desarrollo de experiencias laborales reales, el manejo de nuevas tecnologías, el desarrollo de nuevas competencias y el fortalecimiento de capacidades de toma de decisiones y autogestión.

Para esto, es fundamental que la academia universitaria fortalezca los programas de vinculación con organizaciones del mundo del trabajo, al menos con las instituciones empleadoras y los colegios profesionales, en el caso de educación, relación que permita conocer, y por qué no, codiseñar los planes de estudio, para asegurar la pertinencia social de la oferta académica formal y no formal, como son los programas de educación permanente, ya sean bajo la modalidad de vinculación externa remunerada o extensión universitaria.

Como resultado de dicha vinculación, podrían resultar colaboraciones importantes que favorezcan ambos a sectores, por ejemplo, la posibilidad de adelantar y curricularizar experiencias laborales para el estudiantado universitario bajo la figura de pasantía laboral, así como proyectos de investigación interinstitucionales e intersectoriales.

\section{ReFerencias Bibliográficas}

Albrieu, R., Brest, C., Larroulet, P., Rapetti, M. y Sorrentino, A. (2019). Inteligencia artificial y crecimiento económico. Oportunidades y desafios para Costa Rica. Buenos Aires, Argentina: Cippec. Recuperado de https://bit.ly/2RW pwrb

Arrieta, E. (07 junio, 2019). "Títulos universitarios pierden importancia”: Carlos Alvarado. La República.net. Recuperado de https://bit.ly/3mLGs1N

Asamblea General de Naciones Unidas. (1989). Convención sobre los Derechos del Niño. Ginebra, Suiza: UNICEF

Asamblea Nacional Constituyente. (1949). Constitución Politica de la República de Costa Rica. San José, Costa Rica: Imprenta Nacional

Banco Mundial. (2017). Trouble in the making. Washington, D.C. Estados Unidos: World Bank Group.

Cordero, M. (2018). Estado de la Educación critica calidad de los docentes. Semanario Universidad. Recuperado de https://bit.ly/3hU1F6m

Echeverría, B., Isus, S., Martínez, M. y Sarasola, L. (2008). Orientación Profesional. Barcelona, España: OUC.

Enriques, M. (2007). Desarrollo y gestión de carreras con adultos en el siglo XXI: lecturas hacia una armonización de lo global y de lo individual. Electronic Journal of Research in Educational Psychology, 5(1), 75-101. Recuperado de https://bit.ly/3kFOxUa

González, F. (2019). Modelos de intervención de los servicios de empleo en las universidades públicas costarricenses: una caracterización teórica desde la orientación laboral. (Tesis de maestría). Universidad de Costa Rica, Costa Rica. Recuperado de https://bit.ly/33VvDSe

Gorz, A. (1997). La metamorfosis del trabajo. Madrid, España: Iniciativas Editoriales Sisrema. S. A.

Gutiérrez, I., Kikut, L., Corrales, K. y Picado, C. (2018). Seguimiento de la Condición Laboral de las Personas Graduadas 2011-2013 de las Universidades Costarricenses. San José, Costa Rica: CONARE.

Gutiérrez, I., Corrales, K. y Sandí, K. (2019). Seguimiento de la condición laboral de las personas graduadas 2011-2013 de la Universidad Nacional. San José, Costa Rica: CONARE. 
Grüner, E. (Coord.) (2011). Nuestra América y el pensamiento crítico. Fragmentos de pensamiento crítico de Latinoamérica y el Caribe. Buenos Aires, Argentina: Clacso.

Hausmann, R. (1919). El espiritu de nuestro tiempo. [Escultura]. París, Francia: Centre Pompidou, Musée national d'art moderne-Centre de création industrielle.

Instituto Nacional de Estadística y Censo (INEC). (2019). Encuesta Continua de Empleo al tercer trimestre de 2019. Resultados generales. San José, Costa Rica: INEC. Recuperada de https://bit.ly/346uoQz

Mészàros, I. (2002). Para além do capital. Sao Paulo, Brasil: Bitempo.

Organización de las Naciones Unidas (ONU). (1948). Declaración Universal de los Derechos Humanos. París, Francia: ONU

Organización de los Estados Americanos (OEA). (1969). Convención Americana sobre Derechos Humanos (Pacto de San José). San José, Costa Rica: OEA.

Organización Mundial del Trabajo. (2019). Perspectivas sociales y del empleo en el mundo: Tendencias 2019. Recuperado de https://www.ilo.org/wcmsp5/groups/public/---dgreports/---dcomm/---publ/documents/publication/wcm s_670569.pdf

Programa Estado de la Nación (2019). Séptimo Informe Estado de la Educación. San José, Costa Rica: Masterlitho. Recuperado de https://bit.ly/3iUm8JB

Rivas, F. (2003). Asesoramiento vocacional: Teoría, práctica e instrumentación. España: Ariel Psicología.

Teichler, U. (2005). Graduados y empleo: investigación metodológica y resultados. Los casos de Europa, Japón, Argentina y Uruguay. Buenos Aires, Argentina: Miño y Dávila SRL.

\section{Notas}

[1] En general, el desempleo profesional es cerca del 5,6\% según el OLaP, y la tasa de ocupación es mayor al 90\%.

[2] "La Educación Superior es un derecho humano y un bien público social. Los Estados tienen el deber fundamental de garantizar este derecho. Los Estados, las sociedades nacionales y las comunidades académicas deben ser quienes definan los principios básicos en los cuales se fundamenta la formación de los ciudadanos y ciudadanas, velando porque ella sea pertinente y de calidad" (Conferencia Regional de Educación Superior, 2008, párr. 9).

[3] Lo absurdo se sustenta en la no relación del ingreso con el éxito, ya que la asignación salarial o el ingreso producto de una actividad productiva no tiene significancia en la aceptación social o incidencia en el corolario social. El sentido social de los seres humanos no radica en el éxito, pues es un constructo imaginario y a conveniencia, de ahí su intrascendencia, al igual que la riqueza individual.

[4] Según el Semanario Universidad, se gradúan cerca de 10.000 docentes al año, y "la mayor sobreoferta fue para puestos de Educación Preescolar con 8247 y Educación Especial con 18.049” (Cordero, 2018, párr. 15).

\section{BY-NC-ND}

